\title{
Comparative Statics Analysis on Cubic Mechanism
}

\author{
Jianguo Luo* \\ College of Mechanical \& Electrical Engineering \\ North China Institute of Science and Technology \\ Beijing, 101601, China \\ *Corresponding author
}

\author{
Maoyan He \\ Labour Union \\ North China Institute of Science and Technology \\ Beijing, 101601, China
}

\author{
Yuanyuan Liu \\ College of Mechanical \& Electrical Engineering \\ North China Institute of Science and Technology \\ Beijing, 101601, China
}

\begin{abstract}
Present a new type of cubic mechanism, which based on the traditional serial manipulators and parallel manipulators, given the position and orientation of the whole mechanism and the output shaft force screw, theory of wrench of multi-rigid body adopted to find the inverse solution of force screw of each input jointed point, analysis carried out according to normal and special conditions, results obtained with practical example setup with program to get the data graph of mechanism, the results of inverse solution of statics analysis obtained sensitively.
\end{abstract}

Keywords-cubic mechanism; rigid body; wrench; inverse solution; programming

\section{INTRODUCTION}

Statics analysis is a necessary part of the research on mechanism, which is the basis of dynamic analysis and rigidity analysis, also as the following work of kinematics analysis. Lu Guanda[1] complete statics analysis on the 3-RSS/S parallel mechanism by component vector method, and force feedback control experiment adopted to verify the static analysis model can be used as a theory basis for force control under low speed. Zhou Yulin[2] established the statics equilibrium equation of the 2-DOF spherical parallel mechanism UP $+R$ through the method of split bars, considering the elasticity of components, deformation coordination equations built by using the principle of small deformation superposition, thus complete the static analysis. Wang Shaofeng[3], Fan Rui[4], Jidai[5] researched on these kind of mechanism and problems, some fruits obtained then. Matlab used to write the program of horizontal axis and state of force, also simulation and calculation analysis carried out. In view of the practical needs of six degree of freedom decoupling mechanism, a new type of hybrid cubic mechanism based on $\mathrm{S}(\mathrm{P})[\mathrm{T}]$ output base and $\mathrm{P}$ input base put forward in this paper, as shown in Figure 1, which can achieve six degree of freedom through three-dimensional linear driving[7]. Screw theory selected to find the wrench of each input joint, intuitive results obtained through analysis and simulation on example.

\section{ANALYSIS ON FORCE SCREW}

Position of input joints and pose of output shaft given, as shown in Figure 2, $\mathrm{F}_{\mathrm{m}}$ is the applied force screw of executor end, $M_{m}$ is the torque in the same direction. Spatial arbitrary force system can be simplified into superposition of force $\boldsymbol{f}_{i}\left(\boldsymbol{S}_{1} ; \boldsymbol{S}_{0}\right)$ and torque $C_{2}\left(0 ; S_{2}\right)$, both meet the relationship of summary in screw algebra, thus we get

$$
\boldsymbol{f}_{\boldsymbol{i}} \boldsymbol{S}_{i}=\boldsymbol{f}_{\boldsymbol{i}} \boldsymbol{S}_{\boldsymbol{i}}+\in \boldsymbol{f}_{\boldsymbol{i}} \boldsymbol{S}_{\boldsymbol{i}}^{0},
$$

$\boldsymbol{S}_{\boldsymbol{i}}$ is the unit vector, $\boldsymbol{\$}_{i}$ is the unit wrench, $\boldsymbol{S}_{\boldsymbol{i}} \cdot \boldsymbol{S}_{\boldsymbol{i}}=1, \boldsymbol{S}_{\boldsymbol{i}}$ orthogonal with $\boldsymbol{S}_{\boldsymbol{i}}^{0}$, there exists $\boldsymbol{S}_{\boldsymbol{i}} \cdot \boldsymbol{S}_{\boldsymbol{i}}^{\boldsymbol{0}}=0$, position and pose of mechanism given, force screw and related parameters provided as well, analysis on force and torque of output shaft $\mathrm{O}_{1} \mathrm{O}_{2}$ and each input joints can be carried out then.

Before the start of analysis, relevant parameters defined, $h$ is the distance between the centers of upper platform and lower platform, $\mathrm{h}$ is the distance between the center of lower platform and output end point $\mathrm{P}, \alpha, \beta, \gamma$ are the direction angle between output shaft and fixed coordinate system, c and $\mathrm{s}$ denote cos and sin respectively(following as the same). Take the center point $O_{1}$ as the pivot point, as shown in Figure 3, the balance equations of torque (2) to (4) listed as following

$$
\begin{aligned}
& F_{m}^{y} \cdot\left|P O_{1}\right| \cdot|c \beta|+F_{m}^{z} \cdot\left|P O_{1}\right| \cdot|c \gamma|+M_{m}^{x}=, \\
& F_{O_{2}}^{z} \cdot\left|O_{1} O_{2}\right| \cdot|c \gamma|+F_{O_{2}}^{y} \cdot\left|O_{1} O_{2}\right| \cdot|c \beta| \\
& F_{m}^{x} \cdot\left|P O_{1}\right| \cdot|c \alpha|+F_{m}^{z} \cdot\left|P O_{1}\right| \cdot|c \gamma|+M_{m}^{y}=, \\
& F_{O_{2}}^{z} \cdot\left|O_{1} O_{2}\right| \cdot|c \gamma|+F_{O_{2}}^{x} \cdot\left|O_{1} O_{2}\right| \cdot|c \alpha| \\
& F_{m}^{x} \cdot\left|P O_{1}\right| \cdot|c \beta|+F_{m}^{y} \cdot\left|P O_{1}\right| \cdot|c \beta|+M_{m}^{z}=, \\
& F_{O_{2}}^{x} \cdot\left|O_{1} O_{2}\right| \cdot|c \alpha|+F_{O_{2}}^{y} \cdot\left|O_{1} O_{2}\right| \cdot|c \beta|
\end{aligned}
$$

Take the center point $\mathrm{O}_{2}$ as the pivot point, as shown in Figure 4, the balance equations of torque (5) to (7) listed as 
following

$$
\begin{aligned}
& F_{m}^{y} \cdot\left|P O_{2}\right| \cdot|c \beta|+F_{m}^{z} \cdot\left|P O_{2}\right| \cdot|c \gamma|+M_{m}^{x}=, \\
& F_{O_{1}}^{z} \cdot\left|O_{1} O_{2}\right| \cdot|c \gamma|+F_{O_{1}}^{y} \cdot\left|O_{1} O_{2}\right| \cdot|c \beta| \\
& F_{m}^{x} \cdot\left|P O_{2}\right| \cdot|c \alpha|+F_{m}^{z} \cdot\left|P O_{2}\right| \cdot|c \gamma|+M_{m}^{y}=, \\
& F_{O_{1}}^{z} \cdot\left|O_{1} O_{2}\right| \cdot|c \gamma|+F_{O_{1}}^{x} \cdot\left|O_{1} O_{2}\right| \cdot|c \alpha| \\
& F_{m}^{x} \cdot\left|P O_{2}\right| \cdot|c \beta|+F_{m}^{y} \cdot\left|P O_{2}\right| \cdot|c \beta|+M_{m}^{z}=, \\
& F_{O_{1}}^{x} \cdot\left|O_{1} O_{2}\right| \cdot|c \alpha|+F_{O_{1}}^{y} \cdot\left|O_{1} O_{2}\right| \cdot|c \beta|
\end{aligned}
$$

When the direction of axis of force and torque on output shaft end coincide with the axis of output shaft, as shown in Figure 5, because the lower platform is Hooke joint, rotation around the axis of output shaft is limited, we just need to consider the force on lower platform, take the center point $\mathrm{O}_{2}$ as the pivot point, the balance equations of torque (8) to (10) listed as $F_{m}^{x}=F_{O_{1}}^{x}, F_{m}^{y}=F_{O_{1}}^{y}, F_{m}^{z}=F_{O_{1}}^{z}$.

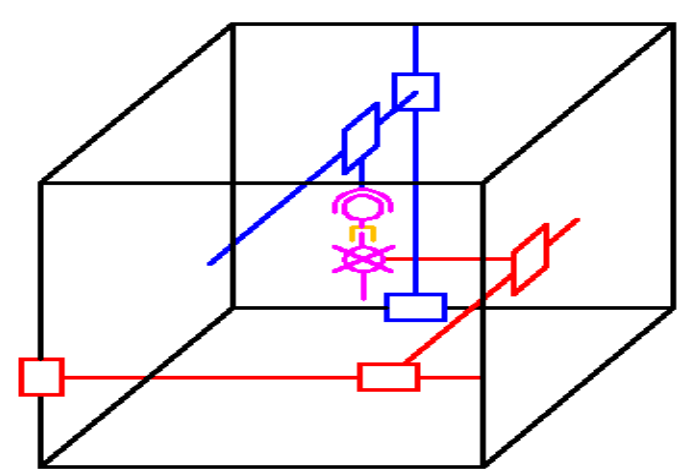

FIGURE I. SCHEMATIC DIAGRAM OF CUBIC MECHANISM

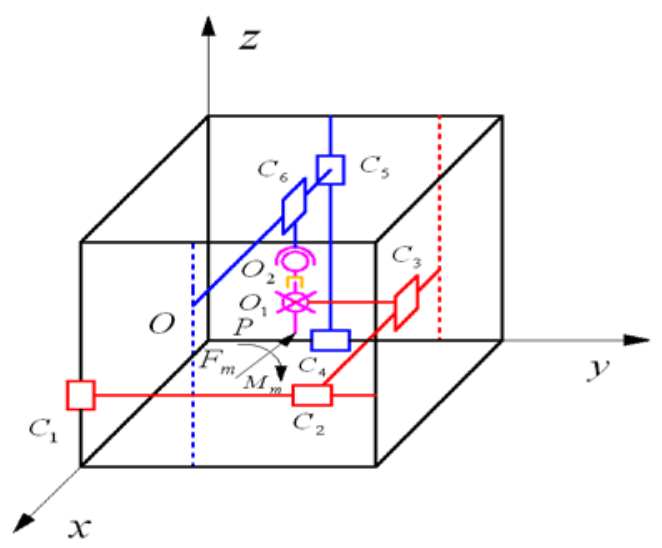

FIGURE II. APPLIED FORCE AND TORQUE ON MECHANISM

We can see from the expression (2) to (7) that six unknown parameters exist in equations (2) to (7). That is the sub force along the axis $\mathrm{x}$ and $\mathrm{y}$ and $\mathrm{z}$, which can all be found through these six equations.
Among them, $F_{O_{1}}^{x}, F_{O_{1}}^{y}, F_{O_{1}}^{z}, F_{O_{2}}^{x}, F_{O_{2}}^{y}, F_{O_{2}}^{z}$ is the sub force along the axis $\mathrm{x}$ and $\mathrm{y}$ and $\mathrm{z}$ of each points on moving platform. Expression (2) to (7) can be written into expression (11), that is $[J] \cdot[U]=[b],[\mathrm{J}]$ is the Jacobian matrix, $[\mathrm{U}]$ is the sub force variable matrix, [b] is a constant matrix, $[U]=\left[\begin{array}{llllll}F_{O_{1}}^{x} & F_{O_{1}}^{y} & F_{O_{1}}^{z} & F_{O_{2}}^{x} & F_{O_{2}}^{y} & F_{O_{2}}^{z}\end{array}\right]^{\prime}$, when the Jacobian matrix is not a singular matrix, the inverse of $[\mathrm{J}]$ exists, thus we get $[U]=[J]^{-1}[b]$.

Wrench on center point $\mathrm{O}_{1}$ and $\mathrm{O}_{2}$ both zero after further analysis, inverse solution of statics of each input points can be obtained then.

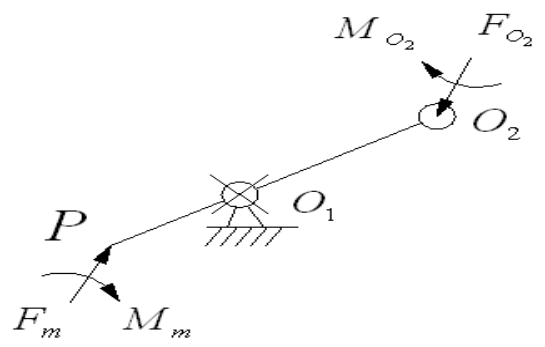

FIGURE III. HORIZONTAL TORQUE ANALYSISOF LOWER PLATFORM AROUND AXIS $\mathrm{O}_{z}$

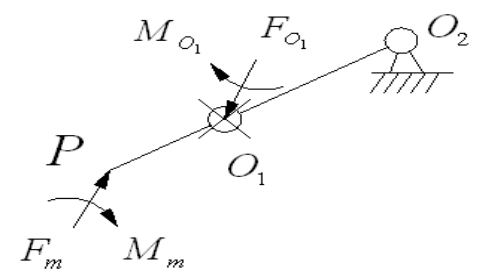

FIGURE IV. ANALYSIS OF SCREW OF OUTPUT SHAFT WITH $\mathrm{O}_{2}$ AS THE PIVOT POINT

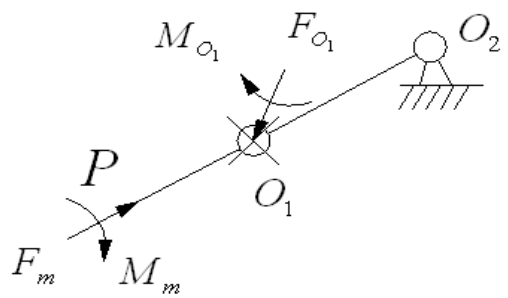

FIGURE V. ANALYSIS OF SCREW OF OUTPUT SHAFT WITH $\mathrm{O}_{2}$ AS THE PIVOT POINT IN SPECIAL CONDITION

According to the balance equations of force screw, force and torque of each input points $C_{i}(i=1,2,3,4,5,6)$ on upper and lower platform can be found quickly. Due to the special structure of the mechanism, following relationship exist between the sub force along the axis of coordinate system(as shown in Figure 2) of each input sliders and the sub force along the axis of coordinate system of center points on upper and lower platform, $F_{C_{1}}^{z}=F_{O_{1}}^{z}, F_{C_{2}}^{y}=F_{O_{1}}^{y}, F_{C_{3}}^{x}=F_{O_{1}}^{x}, F_{C_{5}}^{z}=F_{O_{2}}^{z}$, 
$F_{C_{4}}^{y}=F_{O_{2}}^{y}, \quad F_{C_{6}}^{x}=F_{O_{2}}^{x}$. Other sub force of each input sliders are zero, the sub torque along the coordinate axis of each input sliders list as following, $M_{C_{1}}^{x}=F_{O_{1}}^{z} \times\left|O_{1} C_{1}^{y}\right|$, $M_{C_{1}}^{y}=F_{O_{1}}^{z} \times\left|O_{1} C_{1}^{x}\right| \quad, \quad M_{C_{1}}^{z}=F_{O_{1}}^{x} \times\left|O_{1} C_{1}^{y}\right|+F_{O_{1}}^{y} \times\left|O_{1} C_{1}^{x}\right| \quad$, $M_{C_{2}}^{x}=F_{O_{1}}^{z} \times\left|O_{1} C_{2}^{y}\right|, \quad M_{C_{2}}^{y}=F_{O_{1}}^{z} \times\left|O_{1} C_{2}^{x}\right|$, $M_{C_{2}}^{z}=F_{O_{1}}^{x} \times\left|O_{1} C_{2}^{y}\right|+F_{O_{1}}^{y} \times\left|O_{1} C_{2}^{x}\right| \quad, \quad M_{C_{3}}^{x}=F_{O_{1}}^{z} \times\left|O_{1} C_{3}{ }^{y}\right|$ $\boldsymbol{M}_{C_{3}}^{y}=\mathbf{O}, M_{C_{3}}^{z}=F_{O_{1}}^{x} \times\left|O_{1} C_{3}{ }^{y}\right|, \quad M_{C_{4}}^{x}=F_{O_{2}}^{y} \times\left|O_{2} C_{4}{ }^{z}\right|$, $M_{C_{4}}^{y}=F_{O_{2}}^{z} \times\left|O_{2} C_{4}{ }^{x}\right|+F_{O_{2}}^{x} \times\left|O_{2} C_{4}{ }^{z}\right|, \quad M_{C_{4}}^{z}=F_{O_{2}}^{y} \times\left|O_{2} C_{4}{ }^{x}\right|$, $M_{C_{5}}^{x}=F_{O_{2}}^{y} \times\left|O_{2} C_{5}{ }^{z}\right|, \quad M_{C_{5}}^{y}=F_{O_{2}}^{z} \times\left|O_{2} C_{5}{ }^{x}\right|+F_{O_{2}}^{x} \times\left|O_{2} C_{5}{ }^{y}\right|$, $M_{C_{5}}^{z}=F_{O_{2}}^{y} \times\left|O_{2} C_{5}{ }^{x}\right| \quad, \quad M_{C_{6}}^{x}=F_{O_{2}}^{z} \times\left|O_{2} C_{6}{ }^{y}\right|$, $M_{C_{6}}^{y}=0, M_{C_{6}}^{z}=F_{O_{2}}^{x} \times\left|O_{2} C_{6}{ }^{y}\right|$.

Among them, $O_{1} C_{i}{ }^{x}$ and $O_{1} C_{i}{ }^{y}$ and $O_{1} C_{i}{ }^{2}(i=1,2,3)$ are the sub vector of center point on lower platform to each slider on it, $\mathrm{O}_{2} \mathrm{C}_{j}{ }^{x}$ and $\mathrm{O}_{2} C_{j}{ }^{y}$ and $\mathrm{O}_{2} C_{j}{ }^{z}(j=4,5,6)$ are the sub vector of center point on upper platform to each slider on it. When the mechanism in the special condition, as shown in Figure 5, there no force and torque exist on the center point of upper platform, force and torque exist only on the center point of lower platform, force and torque of each input sliders on lower platform can be obtained according to the method in normal condition, shown as expression, $F_{C_{1}}^{Z}=F_{m} \cdot c \gamma$, $F_{C_{2}}^{y}=F_{m} \cdot c \beta, F_{C_{3}}^{x}=F_{m} \cdot c \alpha$. Torque of each input sliders on lower platform can be obtained from expression (12) to (20), torque of each input sliders on upper platform are zero.

\section{EXAMPLE ANALYSIS}

Related parameters of mechanism given as follows, $\mathrm{a}=1.0 \mathrm{~m}, \quad\left|\mathrm{PO}_{1}\right|=0.1 \mathrm{~m},\left|\mathrm{O}_{1} \mathrm{O}_{2}\right|=0.2 \mathrm{~m},\left|\mathrm{O}_{1} C_{3}\right|=0.1 \mathrm{~m}$, $\left|\mathrm{O}_{2} \mathrm{C}_{6}\right|=0.1 \mathrm{~m}$. Assume the vertical height in cubic mechanism of output shaft end point $\mathrm{P}$ is $0.3 \mathrm{~m}$. Point $S:(500,500,300)$ as the center of circle, circular motion with a radius of $0.2 \mathrm{~m}$ around vertical coordinate axis $\mathrm{Oz}$ in the horizontal plane, cosine angle between output shaft $\mathrm{O}_{1} \mathrm{O}_{2}$ and coordinate axis

$\mathrm{Oz}$ always keep $30^{\circ}, \mathrm{Fm}=100 \mathrm{~N}, \mathrm{Mm}=100 \mathrm{Nm}$, the direction of force and torque is [1 11 1]. Matlab used to program and simulate, results of force and torque of each input sliders in normal condition obtained then, as shown in Figure 6. If the applied force and torque meet the special condition(as shown in Figure 5), the force and torque of each input sliders on lower platform will be shown in Figure 7.

\section{CONCLUSION}

Following conclusion can be drawn by analyzing and comparing the force and torque of each input joints of the mechanism when the output shaft moving along the given trajectory. In normal condition, the force of each input points on upper and lower platform always keep constant, except the torque at point $C_{3}$ and $C_{6}$ always keep constant, the rest of other input points maintain linear change. In special condition, the force and torque of each input points on upper platform are always zero, the force and torque of each input points on lower platform are not zero. In special condition, the force at point $C_{1}$ remains unchanged, the remaining two points show a linear relationship. The torque of point $C_{3}$ changes linearly, the remaining two points showing nonlinear relationship.
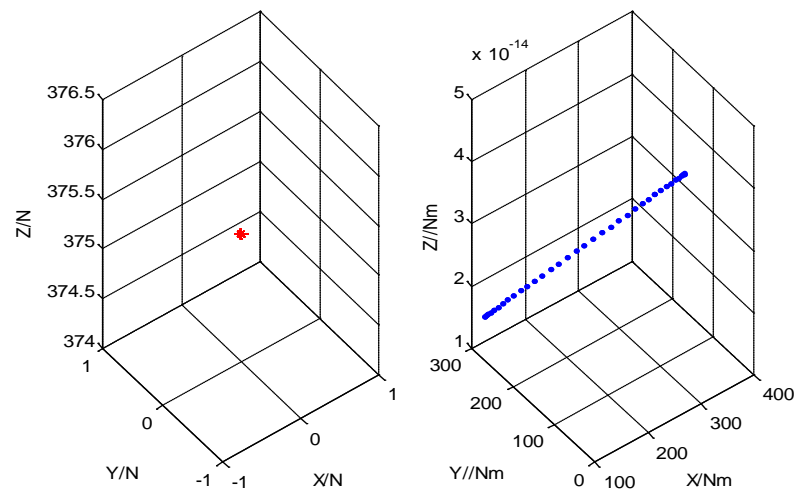

(a) force and torque at point $C_{1}$
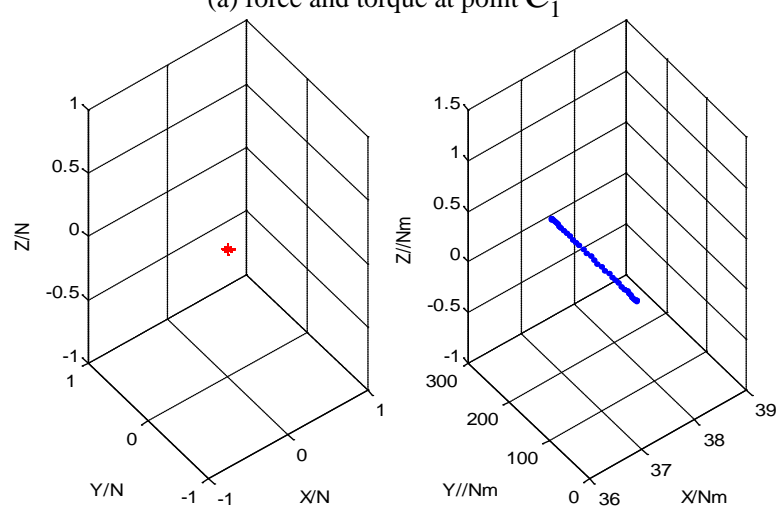

(b) force and torque at point $C_{2}$
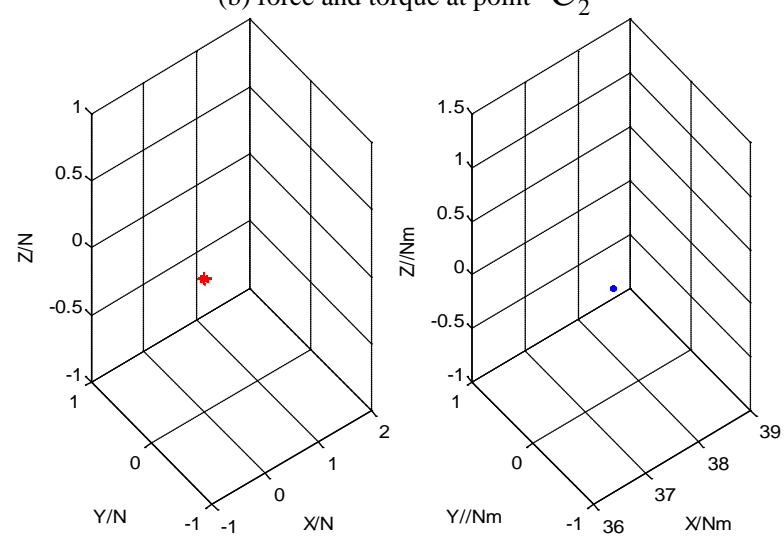

(c) force and torque at point $C_{3}$ 

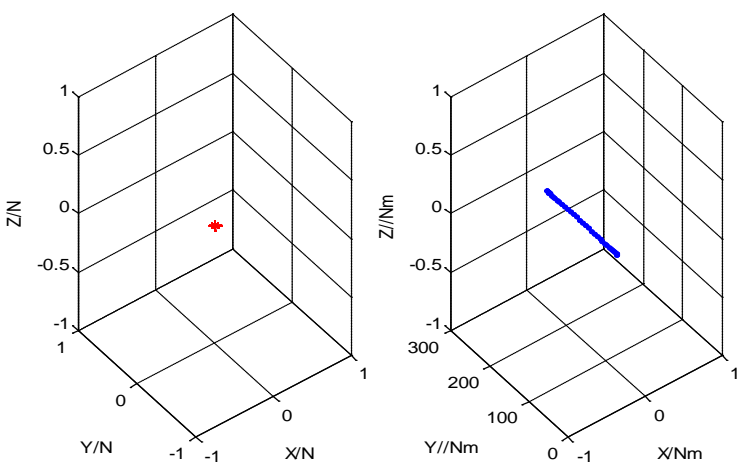

(d) force and torque at point $C_{4}$
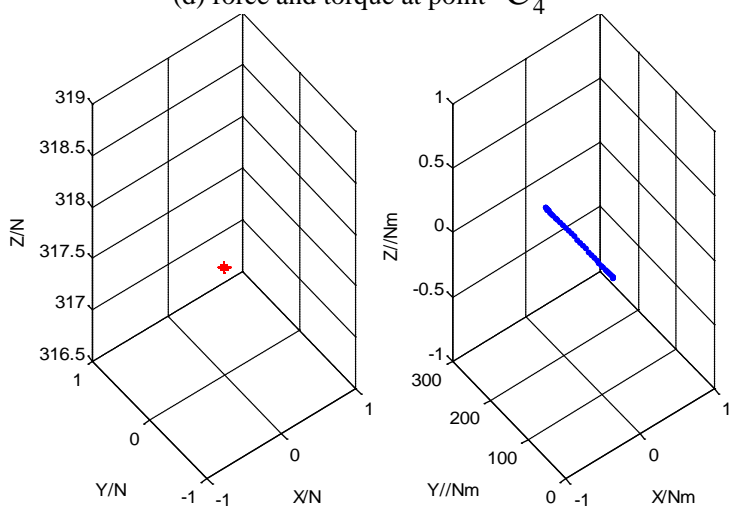

(e) force and torque at point $C_{5}$
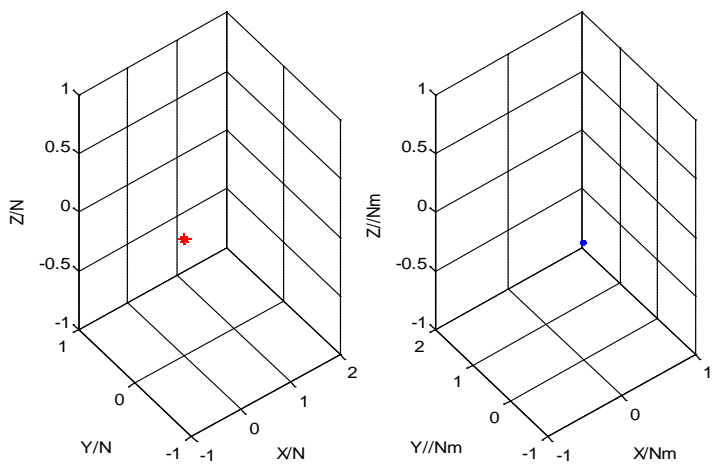

(f) force and torque at point $C_{6}$

FIGURE VI. FORCE AND TORQUE OF POINTS ON UPPER AND LOWER PLATFORM IN NORMAL CONDITION
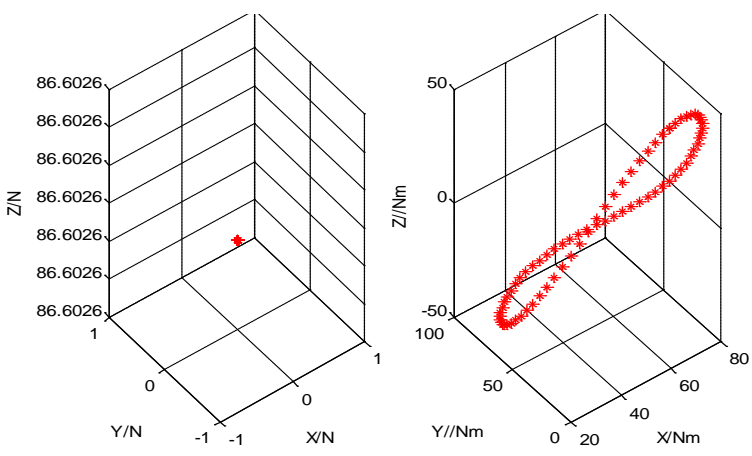

(a) force and torque at point $C_{1}$
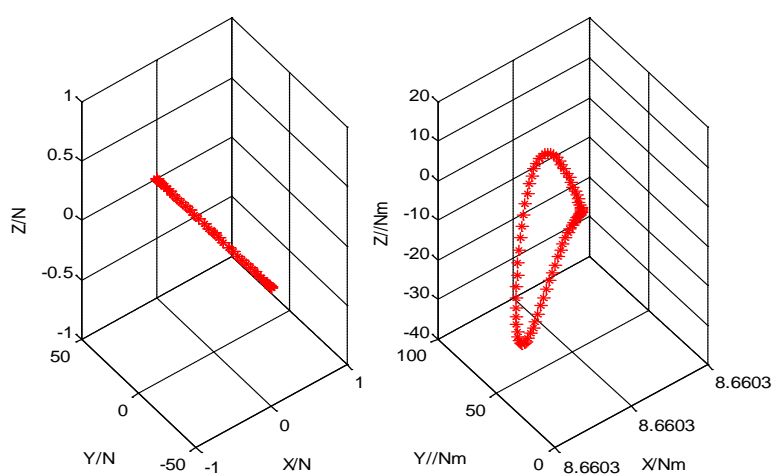

(b) force and torque at point $C_{2}$
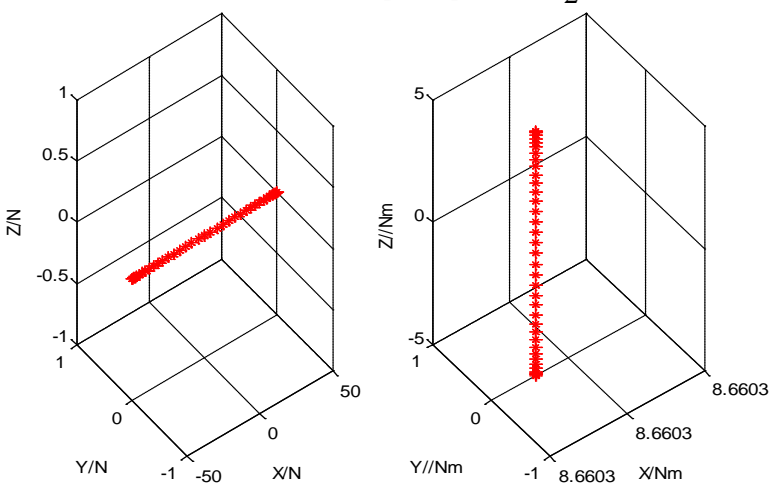

(c) force and torque at point $C_{3}$

FIGURE VII. FORCE AND TORQUE OF POINTS ON LOWER PLATFORM IN SPECIAL CONDITION

\section{ACKNOWLEDGEMENT}

This research was financially supported by the HeBei Province Science and Technology Foundation (No.Z2015072) and NCIST Foundation(No. 3142015023).

\section{REFERENCES}

[1] G. D. Lu, A. M. Zhang, "statics analysis and experimental study on 3-RSS/S parallel mechanism,” Machine design, 2013, Vol.30, pp.26-31.

[2] Y. L. Zhou, B. Li, and L. Yang, "Static analysis on two degree of freedom spherical mechanism U(P+R)," Chinese Mechanical Engineering, 2013, Vol.24, pp. 2081-2087.

[3] S. F. Wang, K. M. Li, "Statics analysis on 3-(2SPS)," Machine tools and hydraulics,” 2013, Vol.41, pp. 55-58.

[4] R. Fan, H. Liu, and D. Wang, "Analysis of statics and stiffness of 3-DOF parallel loading mechanism,” Journal of Beijing university of aeronautics and astronautics, 2014, Vol.40, pp.861-866.

[5] J. D. Wang, F. F. Xin, "Dynamic statics analysis of four degree of freedom hybrid palletizing robot," Mechanical transmission, 2014, Vol.38, pp.76-78. 